ARTICLE

DOI: $10.1038 /$ s41467-018-06608-0

\title{
Contact angle measurement of free-standing square-millimeter single-layer graphene
}

\author{
Anna V. Prydatko', Liubov A. Belyaeva', Lin Jiang ${ }^{1}$, Lia M.C. Lima ${ }^{1}$ \& Grégory F. Schneider ${ }^{1}$
}

Square millimeters of free-standing graphene do not exist per se because of thermal fluctuations in two-dimensional crystals and their tendency to collapse during the detachment from the substrate. Here we form millimeter-scale freely suspended graphene by injecting an air bubble underneath a graphene monolayer floating at the water-air interface, which allowed us to measure the contact angle on fully free-standing non-contaminated graphene. A captive bubble measurement shows that free-standing clean graphene is hydrophilic with a contact angle of $42^{\circ} \pm 3^{\circ}$. The proposed design provides a simple tool to probe and explore the wettability of two-dimensional materials in free-standing geometries and will expand our perception of two-dimensional materials technologies from microscopic to now millimeter scales.

\footnotetext{
${ }^{1}$ Faculty of Science, Leiden Institute of Chemistry, Leiden University, Einsteinweg 55, 2333CC Leiden, The Netherlands. These authors contributed equally: Anna V. Prydatko, Liubov A. Belyaeva. Correspondence and requests for materials should be addressed to G.F.S. (email: g.f.schneider@chem.leidenuniv.nl)
} 
$\mathrm{T}$ he wetting properties of graphene have been a subject of intensive theoretical and experimental investigations over the last decade. Extremely thin and electrically conductive, graphene is widely used in biosensors, lab-on-a-chip and microfluidics platforms where graphene is in contact with water, vapor and analytes ${ }^{1-4}$. Although graphene was long believed to be a graphite-like material ${ }^{5-7}$, some recent studies have shown a wide spread of water contact angle (CA) on graphene $e^{5,6,8-10}$, with values ranging from $10^{\circ}$ when supported by water ${ }^{10}$ to $127^{\circ}$ on solid substrates ${ }^{6}$. One reason for such discrepancies in the values of the contact angle is the difference in sample preparation and measurement conditions ${ }^{5,8}$. The adsorption of airborne hydrocarbons, the cleanliness and quality of the graphene-substrate and graphene-water interface can have significant effects on the measured contact angles, which, however, can be minimized in most cases by conducting experiments in controlled atmospheres and by avoiding the use of polymers during the transfer process $^{11-14}$.

The wetting characteristics of a material are dictated by both the surface and the bulk properties of the material, which implies the impossibility to determine the intrinsic wetting properties of two-dimensional (2D) materials which have no bulk. In other words, all wetting characteristics of graphene, such as contact angle and surface energy, refer not only to the graphene surface but also to the bulk phase underneath it and must not be regarded as solely graphene's properties.

In this respect, probing the wetting characteristics of freestanding graphene can give an indispensable insight for understanding graphene's wettability. Yet, due to the extreme fragility of graphene and other 2D materials, studies on free-standing graphene have been limited to theoretical predictions with only a few experimental works on partially suspended graphene ${ }^{15,16}$. Being the only experimental indication of free-standing graphene's wettability up to now, the contact angle of partially suspended graphene is still an indirect measure and requires multistep sample preparation which may result in an ill-defined graphene-substrate interface yielding a range of contact angle values.

In this work, we present a simple and clean captive bubble design for measuring directly the wettability of free-standing graphene. The captive bubble method, i.e., the injection of an air bubble underneath graphene floating on water, allows for the formation of a graphene free-standing area as large as 1.5 by $1.5 \mathrm{~mm}$, the largest free-standing area that has been reported so far for a 2D material. Essentially, graphene remains floating on the water surface after copper etching, intrinsically preventing any transfer- or handling-related contamination and corrugation. An additional advantage is that the graphene side on which the contact angle is measured (i.e., the side that initially faces copper and then water) has never been exposed to ambient air and is therefore not subjected to airborne hydrocarbons adsorption ${ }^{16,17,18}$.

\section{RESULTS}

Captive bubble versus sessile drop. The captive bubble method measures the wetting contact angle using an air bubble at a solid-liquid interface. Often, the method works best for hydrophilic substrates in which liquid spreads out yielding more difficulties to determine the contact angle with the sessile drop technique, e.g., for contact lenses and hydrogels ${ }^{19,20}$. The captive bubble and sessile drop configurations represent the same threephase equilibrium and, therefore, are equivalent (Fig. 1a, b).
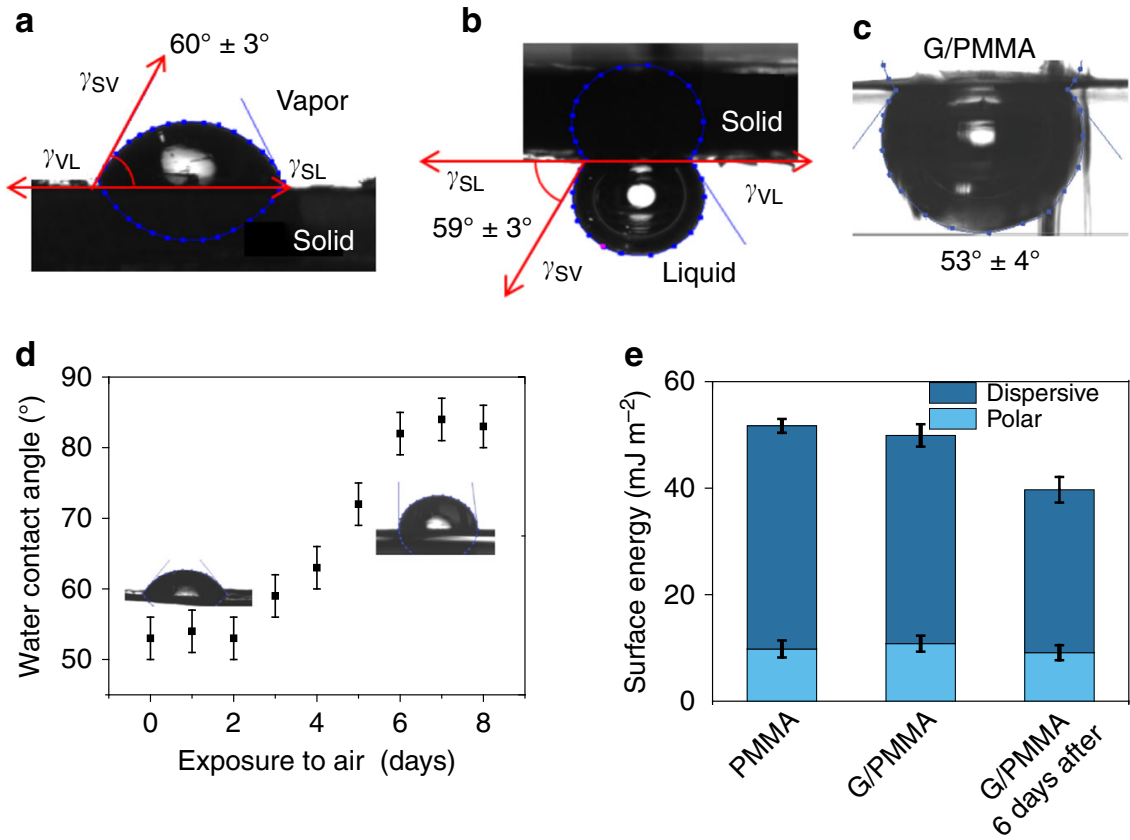

Fig. 1 Sessile drop and captive bubble measurements of graphite and supported graphene. a Sessile drop of water on freshly exfoliated highly oriented pyrolytic graphite (HOPG) in air, where $\gamma_{V L}, \gamma_{S V}$, and $\gamma_{S L}$ are the liquid-vapor, solid-vapor, and thesolid-liquid interfacial tensions, respectively. The measured contact angle is $60^{\circ} \pm 3^{\circ}$. The measurement was reproduced on ten samples and the error bar represents the standard deviation. $\mathbf{b}$ Captive bubble configuration on freshly exfoliated HOPG in water. The measured contact angle is $59^{\circ} \pm 3^{\circ}$. The measurement was reproduced on ten samples and the error bar represents the standard deviation. c Captive bubble measurement of water contact angle on graphene supported by a poly (methyl methacrylate) (PMMA) layer. The measurement was reproduced on ten samples and the error bar represents the standard deviation. $\mathbf{d}$ Sessile drop contact angle measurement of graphene supported by a PMMA layer constantly exposed to air as a function of air exposure time. The measurement was reproduced on three samples and the error bar represents the standard deviation. e Surface energies and polar and dispersive components of the surface energy for PMMA, graphene supported by PMMA, and graphene supported by PMMA which was exposed to air for six days. The measurement was reproduced on three samples and the error bar represents the standard deviation 
The difficulty of the contact angle measurement on freestanding graphene is that $2 \mathrm{D}$ materials do not withstand the mechanical disturbances originating from-for example-depositing a droplet of liquid on their surface because of their extreme thinness ${ }^{10}$. Additionally, free-standing graphene as big as a macroscopic droplet does not exist. Instead, using the captive bubble geometry (i.e., a water-graphene-air bubble interface), allows for a reliable contact angle measurement. Advantages of this method in comparison with the sessile drop technique is that deionized water is primarily composed of water molecules (and therefore less contamination per volume percent compared to air and vacuum; i.e., water protects graphene from airborne hydrocarbon contamination). Another remarkable advantage of the technique is that the bubble is saturated with water, therefore yielding a contact angle in equilibrium in time.

For the comparison of the captive bubble method with the sessile drop technique the water contact angle was measured on highly oriented pyrolytic graphite (HOPG). HOPG was exfoliated with the scotch tape in air or in water depending on the method of contact angle measurement. The average contact angles are $59^{\circ} \pm 3^{\circ}$ for the sessile drop method and $60^{\circ} \pm 3^{\circ}$ for the captive bubble method (Fig. 1a, b). Both methods show high repeatability on solid substrates.

Additionally, contact angles of graphene with a $300 \mathrm{~nm}$ layer of poly(methyl methacrylate) (PMMA) were measured using the captive bubble method and the sessile drop technique as control tests respectively. Graphene appeared wetting transparent in both cases displaying the contact angles of the bare PMMA supportthat is, $53^{\circ} \pm 4^{\circ}$ measured by the captive bubble method (Fig. 1c) and $54^{\circ} \pm 3^{\circ}$ using the sessile drop method (Fig. 1d). Noteworthy, after two days the graphene/PMMA sample became more hydrophobic and after six days the contact angle of graphene increased up to $85^{\circ}$ (Fig. 1d). Such transition from a hydrophilic to a hydrophobic surface is known to be caused by the adsorption of hydrocarbons from the air ${ }^{11}$. A surface-energy analysis using the Owens-Wendt method (Supplementary Note 1) showed that while the graphene surface is clean, hydrocarbons tend to adsorb to minimize the free surface energy. The decrease of the total surface energy and its dispersive component is consistent with previous reports (Fig. 1e) ${ }^{21}$.

The agreement between the sessile drop and captive bubble results for freshly exfoliated graphite and graphene/PMMA in which graphene was not exposed to air shows that, although the air in the bubble may contain hydrocarbon contaminants, they do not affect the contact angle because of the short-lived graphenebubble contact and/or negligible amount of hydrocarbons present in the bubble.

Captive bubble method to study graphene: inflection of floating graphene. For contact angle measurement on graphene using the captive bubble technique, an air bubble is deposited using an inverted needle underneath graphene (Fig. 2a, b; for technical details on the sample preparation and contact angle measurements see the section Methods). From the optical image, one can see that the area of graphene surrounded by air on both sides is 1.5 by $1.5 \mathrm{~mm}$ large, the largest free-standing graphene area ever reported (Fig. 2c).

However, due to its extreme flexibility and thinness, graphene inflects above the surface of water under the pressure of the air bubble and the inflection should be taken into account for the calculation of the contact angle. For flexible materials, forces at the three-phase contact line cannot be described by the Young equation, as it is for flat rigid substrates. Instead, numerous investigations of the contact angle show that the force balance on soft materials is best described by the Neumann's triangle $22-28$. According to Neumann's theory the total contact angle on a deformed substrate can be described as a sum of two angles, beneath and above the contact line, i.e., $\theta_{\text {above }}+\theta_{\text {below }}$ (Fig. $2 \mathrm{~d}$ ). Since the angle measured using the captive bubble method is the contact angle between the air bubble and the solid, i.e., $\theta_{\text {air }}$ the water contact angle should be recalculated as $180^{\circ}-\theta_{\text {air }}$ Taking into consideration the inflection of graphene, the contact angle of water on deflective graphene is therefore $180^{\circ}-\left(\theta_{\text {above }}+\theta_{\text {below }}\right)$ (Fig. 2d).

The measurements of the contact angle of an air bubble on graphene, thus, are more complex than measuring the contact angle
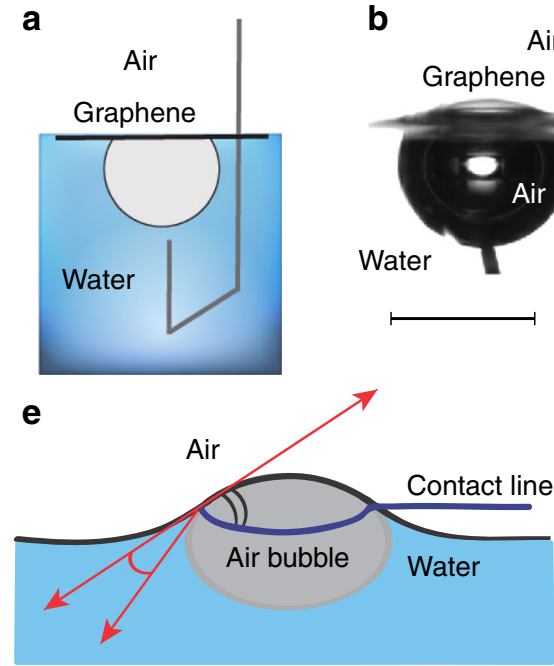

b

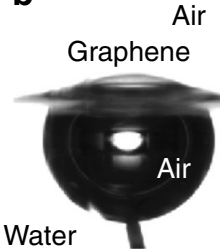

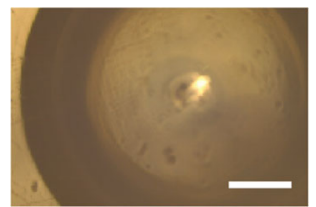

d

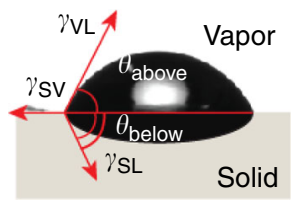

f

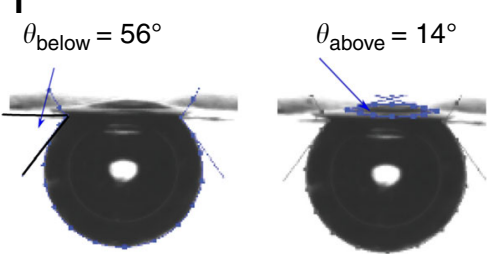

Corrected CA $42^{\circ}$ g

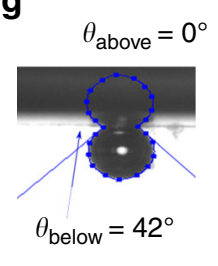

Corrected CA $42^{\circ}$

Fig. 2 Captive bubble configuration for measuring the contact angle of water on free-standing graphene. a Schematic illustration of the captive bubble setup for measuring the contact angle of water on free-standing graphene. $\mathbf{b}$ Optical image of graphene on top of an air bubble (side view). Scale bar represents $2 \mathrm{~mm}$. c Optical image of graphene suspended above the air bubble (top view). Scale bar represents $500 \mu \mathrm{m}$. $\mathbf{d}$ Geometry of the contact line on a soft elastic substrate. The contact angle of three phases is a sum of angles below $\left(\theta_{\text {below }}\right)$ and above $\left(\theta_{\text {above }}\right)$ the horizontal line. e Neumann's triangle. Surface-energy balance for captive bubble on graphene. $\mathbf{f}$ Optical images of a captive bubble on graphene and calculation of the contact angle for an inflected graphene with an air bubble of $6 \mu$ l. $\mathbf{g}$ Optical image of a captive bubble on graphene and calculation of the contact angle (bubble volume $0.2 \mu \mathrm{l}$ ) 
of a drop of water on graphene, and consist of measuring the contact angle measurement above and below the three-phase contact line. The schematic and optical images of an example of a measurement of the contact angle of water on graphene are shown in Fig. $2 e$ and $f$, respectively. The results show that graphene is hydrophilic with a contact angle of water of $42^{\circ} \pm 7^{\circ}$ (Fig. 2f). A video of an air bubble underneath graphene is provided in the Supplementary information (Supplementary Movie 1).

Interestingly, a smaller bubble causes a decrease of the measured angle $180^{\circ}-\theta_{\text {below }}$ and of the inflection angle $\theta_{\text {above }}$, but the difference between the two, i.e., the actual contact angle, is independent of the bubble volume and equal to $42^{\circ} \pm 3^{\circ}$ : for a bubble volume of $6 \mu \mathrm{l}$, the resulting contact angle is $42^{\circ}$ (i.e., the difference between the measured angle of $56^{\circ}$ and the inflection angle of $14^{\circ}$ ), and for a bubble volume of $0.2 \mu$, the measured angle is $42^{\circ}$ and there is no observable inflection to account for as the smaller bubble does not induce significant stretch in the graphene sheet (Fig. 2f, g). These observations are in agreement with other reported works and hypothesis that the sizedependence of the contact angle occurs only on rough and heterogeneous surfaces and not on smooth homogeneous surfaces like graphene ${ }^{29-31}$.

Few-layer graphene and modified graphene. Multilayered graphene (bi- and four-layer) did not exhibit appreciable difference in the water contact angle (Fig. 3a). Since defects and chemisorption of atomic hydrogen/oxygen on graphene are known to affect wetting ${ }^{32}$, we also measured contact angles of graphene modified with $\mathrm{H}_{2}$ and $\mathrm{O}_{2}$ plasma (Fig. 3a, see Methods for details on plasma treatment). After modification with $\mathrm{H}_{2}$ plasma the contact angle on graphene-on-copper decreased from $76^{\circ} \pm 5^{\circ}$ to $68^{\circ} \pm 5^{\circ}$, which can be explained by the cleaning effect of the plasma $^{33,34}$ (Raman characterization of graphene before and after the modification, Supplementary Figure 1). We did not notice a difference in the wettability of suspended graphene after the surface modification with a $\mathrm{H}_{2}$ plasma. Separately, an air bubble on graphene modified by a $\mathrm{O}_{2}$ plasma was very unstable and tended to slip away from the graphene which could be explained by oxygen functionalities induced by the $\mathrm{O}_{2}$ plasma ${ }^{35}$. Overall, contact angle values of modified and multilayer graphene are equal to the contact angle of monolayer pristine graphene given the error margins (Fig. 3a).

Effect of humidity. Recently graphene has been shown to turn hydrophilic when floating on water due to the wetting transparency effect ${ }^{10,36}$. In order to test the effect of the environment on

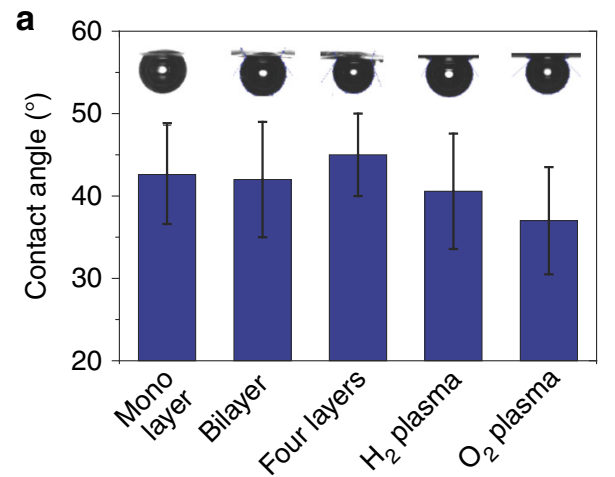

one side of graphene on its hydrophobicity on the other side, we performed the experiments under different humidities. We measured contact angles of water using the captive bubble method with humidities of $98 \%, 85 \%$, and $50 \%$ regulated by saturated salt solution of $\mathrm{K}_{2} \mathrm{SO}_{4}$ and $\mathrm{KCl}^{37}$ (see Methods for more details). Interestingly, a higher humidity level yields lower water contact angle: $29^{\circ} \pm 8^{\circ}$ at $98 \%$ humidity and $42^{\circ} \pm 7^{\circ}$ at $50 \%$ humidity (Fig. 3b).

As for all microscopic approaches, the captive bubble method is technically challenging for studying $2 \mathrm{D}$ materials because they are fragile and even small vibrations can break them apart. In some cases, cracks and holes appear on the graphene surface during the etching process ${ }^{15}$, which can prevent an air bubble from staying underneath the graphene. Furthermore, CVD (chemical vapor deposition) grown graphene is not monocrystalline and has grain boundaries, which could make graphene permeable to air ${ }^{38,39}$. In our experiments, the air bubble underneath graphene was stable from two seconds up to fifteen minutes after which either the bubble or graphene would collapse (Supplementary Figure 2 for optical images before and after the captive bubble experiment). We found that the main sources of the degradation of graphene quality when floating on water are high rate of copper etching, vibrations, intense air circulation and, partly as a result of all the aforementioned, movability of the graphene on the surface of water. In fact, the quality and stability of the floating graphene was significantly improved by using a less concentrated etchant solution of ammonium persulfate $(0.3 \mathrm{M}$ and lower), or by minimizing vibrations and air circulations, and, importantly, immobilizing graphene with a lipid clamp ${ }^{40}$ (see Methods for more details on the lipid clamp and sample preparation).

\section{Discussion}

Although partly suspended graphene on a texturized substrate shows hydrophobic properties with contact angle up to $85^{\circ 15,16}$, our findings demonstrated that clean fully free-standing graphene is mildly hydrophilic (with a measured water contact angle of $42^{\circ}$, in agreement with theoretical predictions on the hydrophilicity of graphene with contact angle of water ranging from $37^{\circ}$ to $44^{\text {o11,17,41 }}$ ). However, such low contact angle is rather surprising, because given the wetting transparency of graphene ${ }^{9,10}$, the contact angle of free-standing graphene should be identical to the contact angle of air, i.e., $180^{\circ}$. The wetting behavior, therefore, in this case cannot be only dictated by the transmission of water-air interactions, but is substantially affected by the phenomena occurring at the graphene surface. Remarkably, the measured contact angle values for mono-, bi-, four-layer graphene, and

b

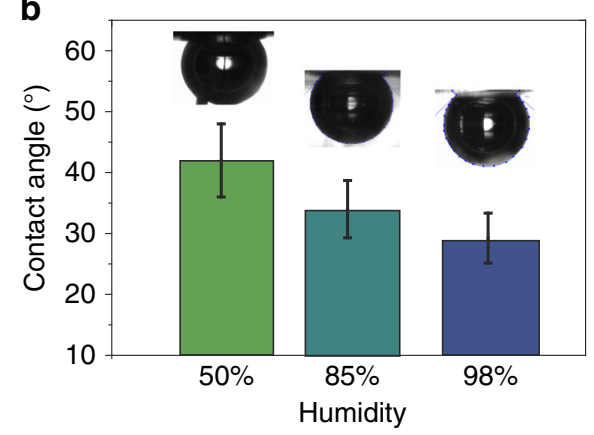

Fig. 3 Graphene contact angles measured by the captive bubble method. a Contact angles of water on free-standing, monolayer, bilayer, four-layer graphene and graphene modified with $\mathrm{H}_{2}$ and $\mathrm{O}_{2}$ plasma, measured using the captive bubble method. The measurement was reproduced on five samples and the error bar represents the standard deviation. b Contact angle of water on free-standing graphene in 50\%, $85 \%$, and $98 \%$ relative humidity. The measurement was reproduced on two samples and the error bar represents the standard deviation 
graphene treated with $\mathrm{O}_{2}$ and $\mathrm{H}_{2}$ plasma are similar (Fig. 3a), also supporting this assumption. The hydrophilicity of graphene (i.e., the fact that water wets free-standing graphene) could be explained by the formation of $\pi$-hydrogen bonding between water molecules and the aromatic system, as it is for benzene-water interaction ${ }^{42,43}$. Another hypotheses attributes the hydrophilicity of graphene to the spontaneous adsorption of $\mathrm{OH}^{-}$ions on graphene surfaces ${ }^{44}$, which could lead to interactions with water and an increase of the repulsive double layer interaction between air (in the bubble) and graphene. As a complementary evidence for the hydrophilic behavior of graphene in water, stable surfactant-free dispersions of graphene have been recently obtained in degassed water ${ }^{44}$. The apparent inability of graphene to form stable aqueous dispersions, which was previously attributed to the hydrophobicity of graphene, is now explained by the adsorption and further coalescence of nanobubbles on the graphene surface.

On the other hand, an increase in the environment humidity, i.e., the concentration of water molecules on the top side of graphene, leads to a decreasing water contact angle value and therefore an increase in the hydrophilicity of graphene (Fig. 3b), indicating that the transparency of graphene to water-water interactions still has a substantial contribution in addition to the water-graphene interactions mentioned above.

In conclusion, we have obtained a millimeter in size-suspended 2D material by simply harvesting surface tension forces at the air-water-graphene-air interface using an air bubble captivated on graphene floating on water.

Direct contact angle measurements have shown that freestanding graphene has hydrophilic properties. Advantageously to other methods, our technique allows to probe the very water-graphene-air interface, in the cleanest way, avoiding any irregularities arising from the transfer and handling processes. The observed hydrophilicity could be explained by the formation of hydrogen bonds which would impact the spontaneous adsorption of water on the graphene surface.

We believe that this work provides a stimulus to further study the still unexplored basic properties of suspended 2D materials, as their surface chemistry, surface energy, compressive and flexural strength, and device interaction at a millimeter-scale level in a free-standing geometry.

\section{Methods}

Materials. Two types of graphene were used: monolayer graphene on a copper substrate provided by Graphenea and graphene grown in a tube oven on a $25 \mu \mathrm{m}$ copper foil at $1035^{\circ}$ according to the procedure described in ref. ${ }^{45}$. Before conducting contact angle experiments, the backside of graphene-on-copper $(\mathrm{G} / \mathrm{Cu})$ was removed with a $\mathrm{O}_{2}$ plasma. Both types of graphene (i.e., Graphenea and homemade) showed the same results for water contact angle measurements.

Multilayered graphene was prepared by repetitive PMMA transfer ${ }^{46}$ of graphene on $\mathrm{G} / \mathrm{Cu}^{47,48}$.

Highly oriented pyrolytic graphite (HOPG, $7 \times 7 \times 0.8-1.8 \mathrm{~mm}$ with mosaic spread $0.8-1.2$ degree) was purchased from NT-MDT.

Sample preparation. CVD graphene on a copper substrate was placed in a $0.3 \mathrm{M}$ water solution of ammonium persulfate (APS) (98\% Sigma-Aldrich). Once the copper foil was etched away, the APS solution was repeatedly replaced with ultrapure water by sequential diluting steps yielding a clean graphene surface without any observable APS crystals ${ }^{45}$. In general, the presence of ions in water has a very small effect on the surface tension of water-in the order of $3 \%$ or lower at the concentration of $0.3 \mathrm{M}^{49-51}$-and, therefore, negligible effects on the measured contact angle. Consequently, and given the precautions we undertook to replace the etching APS solution by water, we assume that possible presence of residual ions had no effect on the contact angle measurements (the contact angle of water on graphene in $0.1 \mathrm{M} \mathrm{FeCl}_{3}$ is equal to the contact angle of graphene in pure water, Supplementary Figure 3).

To place a $6 \mu \mathrm{l}$ air bubble under the water-graphene-air interface, air was injected through a J-shaped inverted needle underneath the graphene (Fig. 2a). The contact angle was then measured at least five times (unless otherwise noted) at the three-phase line interface (Fig. 2d).
To improve the stability of graphene on the water surface, graphene was surrounded with a Langmuir-Blodgett film of 1,2-dipalmitoyl-sn-glycero-3phosphocholine (DPPC) lipids (Avanti Polar Lipids Inc.) at a surface pressure of $30 \mathrm{mN} \mathrm{m}^{-1}$ as it is described in refs. ${ }^{40,52}$. The lipids had a concentration of $1 \mathrm{mg}$ $\mathrm{mL}^{-1}$ and were first dissolved in $\mathrm{CHCl}_{3} / \mathrm{CH}_{3} \mathrm{OH} 3: 1 \mathrm{vol} \%$. First, graphene on copper $^{45}$ (copper facing down) was placed floating on the etchant solution and the appropriate amount of lipids (depending on the size of the graphene and of the cuvette) was added on the surface of the etchant solution around graphene. The etchant solution was then sequentially replaced with ultrapure water and the contact angle was measured. The lipids are known to only spread on the surface of water (around the graphene) without adsorbing on its surface (as measured by infrared spectroscopy $)^{40}$.

Graphene surrounded with lipids showed a higher stability during the deposition of the air bubble. Both graphene samples, without and with lipids, showed similar measured contact angles, i.e., $42^{\circ} \pm 3^{\circ}$ and $42^{\circ} \pm 3^{\circ}$ respectively (Supplementary Figure 4 and Supplementary Movies 1 and 2), confirming the absence of lipids on the graphene surface.

Immobilizing the graphene with lipids is essential for contact angle measurement. If graphene is not stabilized with lipids, the action of placing the air bubble creates a momentum and pushes the graphene sheet away from the field of view of the camera, despite the fact that the bubble is stable and does not collapse (Supplementary Movie 3).

For contact angle measurements of hydrogenated and oxygenated graphene, graphene was first hydrogenated (respectively, oxygenated) using a $\mathrm{H}_{2}$ (respectively, $\mathrm{O}_{2}$ ) plasma in a computer controlled Diener plasma generator for $247 \mathrm{~s}(1 \mathrm{mbar}, 10 \mathrm{~W})^{34}$

Raman spectroscopy. The quality and the number of layers of all graphene samples were characterized by Raman spectroscopy ${ }^{53}$ at room temperature using a $100 \times$ objective and $457 \mathrm{~nm}$ and $532 \mathrm{~nm}$ lasers at a power below $2 \mathrm{~mW}$ to avoid excessive thermal damage of graphene. Figure S1 displays typical Raman spectra of graphene on copper (a) and transferred onto a $\mathrm{Si} / \mathrm{SiO}_{2}$ wafer (b). The shape of the 2D peak $\left(\sim 2700 \mathrm{~cm}^{-1}\right)$, that can be fitted with a single Lorentzian component is indicative of single-layer graphene ${ }^{53}$. The absence of a D peak at $\sim 1370 \mathrm{~cm}^{-1}$ (Figure S1a, b) suggests a low density of defects for non-treated graphene samples ${ }^{53}$.

For hydrogenated and oxygenated graphene, however, the appearance of the D peak (Figure S1c, d) results from the introduced $s p^{3}$ defect sites ${ }^{34}$. Particularly, the ratio $I(2 \mathrm{D}) / I(\mathrm{G})$ decreased from $\sim 2$ (pristine graphene) to $\sim 1$ after $4 \mathrm{~min}$ of hydrogen plasma treatment, indicating the effective doping in the lattice induced by hydrogenation ${ }^{54}$. Moreover, the appearance of a $\mathrm{D}^{\prime}$ peak $\left(\sim 1620 \mathrm{~cm}^{-1}\right)$ in hydrogenated graphene is also related to the activation of defects. The $I(\mathrm{D}) / I\left(\mathrm{D}^{\prime}\right)$ value of $\sim 10$ further confirms the $s p^{3}$ nature of hydrogenated defects ${ }^{55}$.

Optical microscopy. Optical images of graphene on water and graphene transferred on silicon wafer were taken with a Leica optical microscope (DM $2700 \mathrm{M}$ ).

Contact angle measurements. Contact angle measurements were conducted with a standard Ramé-Hart 250 goniometer (Netcong, NJ) and recorded with the DROPimage advanced v 2.8 software under ambient conditions $\left(22^{\circ} \mathrm{C}\right)$. Two methods were used for the characterization of wetting. For the sessile drop technique a water droplet of 5-7 $\mu \mathrm{L}$ was deposited on a substrate and contact angle was measured within five seconds. For the captive bubble method, an air bubble with a volume of $6 \mu \mathrm{L}$ was supplied with a microsyringe at the interface with an inverted needle (28 gauge, 304 SS Ramé-Hart). The analysis of contact angles from recorded videos were made with the software ImageJ (Drop snake analysis).

Measurements at different humidities. Experiments with controlled humidities were carried out using the saturated salt solution method, commonly used for accurate humidity control and the calibration of hygrometers ${ }^{56-59}$. For that, an oversaturated salt solution is placed in a closed box and certain equilibrium vapor pressure (and thus relative humidity) is created. The oversaturation of the solution assures that the built vapor pressure is stable to presence of moisture sources and sinks (the excess of the salt precipitates and the solution remains saturated with the vapor pressure unchanged) and, therefore, provides a precise humidity level. Different salts have different saturated vapor pressures at a given temperature, and the humidity thus can be varied by changing the chemical composition of the salt.

For our experiments, we used oversaturated solutions of $\mathrm{KCl}$ for the humidity of $85.11 \pm 0.29 \%{ }^{37}$ and $\mathrm{K}_{2} \mathrm{SO}_{4}$ for the humidity of $97.59 \pm 0.53 \%{ }^{37}$. For measurements at every given humidity, a beaker with the corresponding salt solution was placed in a sealed glass chamber with an embedded syringe (for further contact angle measurements) together with the cuvette containing graphene floating on water. Then the contact angle was measured using the captive bubble method. The relative humidity of $50 \%$ was the standard ambient humidity of the laboratory maintained by a moisture extractor and measured by a hygrometer, and the contact angle measurements were conducted without salt solutions.

Surface-energy calculation. The surface energy and its components were calculated from the contact angle measurements of different liquids on target surfaces 
using the Owens-Wendt technique ${ }^{59}$. Ultrapure water, ethanol (96\%), ethylene glycol (99,8\%), diiodomethane (99\%), and methylnaphthalene (95\%) were used as test liquids. Details on measured contact angles, surface tension of liquids and surface-energy calculation are provided in the Supplementary information (Supplementary Note 1 and Supplementary Tables 1 and 2).

\section{Data availability}

All data supporting the findings of this study are available within the article and its Supplementary Information. All other data are available from the corresponding author upon reasonable request.

Received: 22 March 2018 Accepted: 11 September 2018 Published online: 10 October 2018

\section{References}

1. Shuo, S. et al. Graphene-based microfluidics for serial crystallography. Lab. Chip. 16, 3082-3096 (2016).

2. Mirsaidov, U. et al. Scrolling graphene into nanofluidic channels. Lab. Chip. 13, 2874 (2013).

3. Chang, J., Zhou, G., Christensen, E. R., Heideman, R. \& Chen, J. Graphenebased sensors for detection of heavy metals in water: a review. Anal. Bioanal. Chem. 406, 3957-3975 (2014).

4. Dan, Y., Lu, Y., Kybert, N. J., Luo, Z. \& Johnson, A. T. C. Intrinsic response of graphene vapor sensors. Nano Lett. 9, 1472-1475 (2009).

5. Raj, R., Maroo, S. C. \& Wang, E. N. Wettability of graphene. Nano Lett. 13, 1509-1515 (2013).

6. Taherian, F., Marcon, V., Van Der Vegt, N. F. A. \& Leroy, F. What is the contact angle of water on graphene? Langmuir 29, 1457-1465 (2013).

7. Shin, Y. J. et al. Surface-energy engineering of graphene. Langmuir $\mathbf{2 6}$ 3798-3802 (2010).

8. Wang, S., Zhang, Y., Abidi, N. \& Cabrales, L. Wettability and surface free energy of graphene films. Langmuir 25, 11078-11081 (2009).

9. Rafiee, J. et al. Wetting transparency of graphene. Nat. Mater. 11, 217-222 (2012).

10. Belyaeva, L. A., van Deursen, P. M. G., Barbetsea, K. I. \& Schneider, G. F. Hydrophilicity of graphene in water through transparency to polar and dispersive interactions. Adv. Mater. 30, 1-7 (2018).

11. Li, Z. et al. Effect of airborne contaminants on the wettability of supported graphene and graphite. Nat. Mater. 12, 925-931 (2013).

12. Kozbial, A., Gong, X., Liu, H. \& Li, L. Understanding the intrinsic water wettability of molybdenum disulfide $\left(\mathrm{MoS}_{2}\right)$. Langmuir 31, 8429-8435 (2015).

13. Kozbial, A., Zhou, F., Li, Z., Liu, H. \& Li, L. Are graphitic surfaces hydrophobic? Acc. Chem. Res. 49, 2765-2773 (2016).

14. Aria, A. I. et al. Time evolution of the wettability of supported graphene under ambient air exposure. J. Phys. Chem. C. 120, 2215-2224 (2016).

15. Ondarçuhu, T. et al. Wettability of partially suspended graphene. Sci. Rep. 6 , 24237 (2016)

16. Zhao, Y. et al. Investigations on the wettability of graphene on a micron-scale hole array substrate. RSC Adv. 6, 1999-2003 (2016).

17. Yiapanis, G., Makarucha, A. J., Baldauf, J. S. \& Downton, M. T. Simulations of graphitic nanoparticles at air-water interfaces. Nanoscale 8, 19620-19628 (2016).

18. Li, J. \& Wang, F. Water graphene contact surface investigated by pairwise potentials from force-matching PAW-PBE with dispersion correction. $J$. Chem. Phys. 146, 054702 (2017)

19. Read, M. L., Morgan, P. B., Kelly, J. M. \& Maldonado-Codina, C. Dynamic contact angle analysis of silicone hydrogel contact lenses. J. Biomater. Appl. 26, 85-99 (2011)

20. Lin, M. C. \& Svitova, T. V. Contact lenses wettability in vitro: effect of surfaceactive ingredients. Optom. Vis. Sci. 87, 440-447 (2010).

21. Kozbial, A. et al. Study on the surface energy of graphene by contact angle measurements. Langmuir 30, 8598-8606 (2014).

22. Style, R. W. et al. Universal deformation of soft substrates near a contact line and the direct measurement of solid surface stresses. Phys. Rev. Lett. 110, 1-5 (2013).

23. Lubbers, L. A. et al. Drops on soft solids: free energy and double transition of contact angles. J. Fluid. Mech. 747, R1 (2014).

24. Metois, J. J. Elastic straining of a thin graphite layer by a liquid droplet or a non-epitaxed Pb crystallite. Surf. Sci. 241, 279-288 (1991).

25. Jerison, E. R., Xu, Y., Wilen, L. A. \& Dufresne, E. R. Deformation of an elastic substrate by a three-phase contact line. Phys. Rev. Lett. 106, 1-4 (2011).

26. Hui, C.-Y. \& Jagota, A. Deformation near a liquid contact line on an elastic substrate. Proc. R. Soc. A Math. Phys. Eng. Sci. 470, 20140085 (2014).

27. Style, R. W. \& Dufresne, E. R. Static wetting on deformable substrates, from liquids to soft solids. Soft Matter 8, 7177-7184 (2012).
28. Nadermann, N., Hui, C.-Y. \& Jagota, A. Solid surface tension measured by a liquid drop under a solid film. Proc. Natl Acad. Sci. USA 110, 10541-10545 (2013).

29. Drelich, J., Miller, J. D. \& Good, R. J. The effect of drop (bubble) size on advancing and receding contact angles for heterogeneous and rough solid surfaces as observed with sessile-drop and captive-bubble techniques. $J$. Colloid Interface Sci. 179, 37-50 (1996).

30. Drelich, J. The effect of drop (bubble) size on contact angle at solid surfaces. J. Adhes. 63, 31-51 (1997).

31. Drelich, J. \& Miller, J. D. The effect of surface heterogeneity on pseudo-line tension and the flotation limit of fine particles. Colloids Surf. 69, 35-43 (1992)

32. $\mathrm{Xu}, \mathrm{Z}$. et al. Reversible hydrophobic to hydrophilic transition in graphene via water splitting induced by UV irradiation. Sci. Rep. 4, 6450 (2015).

33. Russo, C. J. \& Passmore, L. A. Controlling protein adsorption on graphene for cryo-EM using low-energy hydrogen plasmas. Nat. Methods 11, 649-652 (2014).

34. Jiang, L., Fu, W., Birdja, Y. Y., Koper, M. T. M. \& Schneider, G. F. Quantum and electrochemical interplays in hydrogenated graphene. Nat. Commun. 9, 793 (2018).

35. Mcevoy, N., Nolan, H., Nanjundan, A. K., Hallam, T. \& Duesberg, G. Functionalization of graphene surfaces with downstream plasma treatments. Carbon 54, 283-290 (2013).

36. Driskill, J., Vanzo, D., Bratko, D. \& Luzar, A. Wetting transparency of graphene in water. J. Chem. Phys. 141, 18C517 (2014).

37. Greenspan, L. Humidity fixed points of binary saturated aqueous solutions. J. Res. Natl Bur. Stand. Sect. A Phys. Chem. 81A, 89 (1977).

38. Huang, P. Y. et al. Grains and grain boundaries in single-layer graphene atomic patchwork quilts. Nature 469, 389-392 (2011).

39. Yu, Q. et al. Control and characterization of individual grains and grain boundaries in graphene grown by chemical vapour deposition. Nat. Mater. 10, 443-449 (2011)

40. Lima, L. M. C., Arjmandi-Tash, H. \& Schneider, G. F. Lateral non-covalent clamping of graphene at the edges using a lipid scaffold. ACS Appl. Mater. Interfaces 10, 11328-11332 (2018).

41. Andrews, J. E., Sinha, S., Chung, P. W. \& Das, S. Wetting dynamics of a water nanodrop on graphene. Phys. Chem. Chem. Phys. 18, 23482-23493 (2016).

42. Suzuki, S. et al. Benzene forms hydrogen bonds with water. J. Chem. Phys. 257, 942-944 (1992).

43. Gierszal, K. P. et al. П-Hydrogen bonding in liquid water. J. Phys. Chem. Lett. 2, 2930-2933 (2011).

44. Bepete, G. et al. Surfactant-free single-layer graphene in water. Nat. Chem. 9, 347-352 (2016)

45. Belyaeva, L. A., Fu, W., Arjmandi-Tash, H. \& Schneider, G. F. Molecular caging of graphene with cyclohexane: transfer and electrical transport. ACS Cent. Sci. 2, 904-909 (2016).

46. Suk, J. W. et al. Transfer of CVD-grown monolayer graphene onto arbitrary substrates. ACS Nano 5, 6916-6924 (2011).

47. Batrakov, K. et al. Flexible transparent graphene/polymer multilayers for efficient electromagnetic field absorption. Sci. Rep. 4, 1-5 (2014).

48. Wu, B. et al. Experimental demonstration of a transparent graphene millimetre wave absorber with $28 \%$ fractional bandwidth at $140 \mathrm{GHz}$. Sci. Rep. 4, 1-7 (2014).

49. Weissenborn, P. K. \& Pugh, R. J. Surface tension of aqueous solutions of electrolytes: relationship with ion hydration, oxygen solubility, and bubble coalescence. J. Colloid Interface Sci. 184, 550-563 (1996).

50. Hård, S. \& Johansson, K. The surface tension of concentrated aqueous solutions of 1.1-electrolytes measured by means of Wilhelmy and laser light scattering methods. J. Colloid Interface Sci. 60, 467-472 (1977).

51. Dutcher, C. S., Wexler, A. S. \& Clegg, S. L. Surface tensions of inorganic multicomponent aqueous electrolyte solutions and melts. J. Phys. Chem. A 114, 12216-12230 (2010)

52. Lima, L. M. C., Fu, W., Jiang, L., Kros, A. \& Schneider, G. F. Graphenestabilized lipid monolayer heterostructures: a novel biomembrane superstructure. Nanoscale 8, 18646-18653 (2016).

53. Ferrari, A. C. Raman spectroscopy of graphene and graphite: disorder, electron-phonon coupling, doping and nonadiabatic effects. Solid State Commun. 143, 47-57 (2007).

54. Das, A. et al. Monitoring dopants by Raman scattering in an electrochemically top-gated graphene transistor. Nat. Nanotechnol. 3, 210-215 (2008).

55. Eckmann, A. et al. Probing the nature of defects in graphene by Raman spectroscopy. Nano Lett. 12, 3925-3930 (2012).

56. Wexler, A. \& Brombacher, W. G. Methods of measuring humidity and testing hygrometers. National Bur. Stand512, 1-18 (1951).

57. Lu, T. \& Chen, C. Uncertainty evaluation of humidity sensors calibrated by saturated salt solutions. Meas. J. Int. Meas. Confed. 40, 591-599 (2007).

58. Carotenuto, A. \& Dell'Isola, M. An experimental verification of saturated salt solution-based humidity fixed points. Int. J. Thermophys. 17, 1423-1439 (1996). 
59. Kaelble, D. H. Dispersion-polar surface tension properties of organic solids. J. Adhes. 2, 66-81 (1970)

\section{Acknowledgements}

The work leading to this article has gratefully received funding from the Leiden University Huygens fellowship, from the exchange program Erasmus+, from the European Research Council under the European Union's Seventh Framework Programme (FP/ 2007-2013)/ERC Grant Agreement n. 335879 project acronym 'Biographene', and the Netherlands Organization for Scientific Research (Vidi 723.013.007). Particularly, the we would like to thank Dr. Edgar Blokhuis for his insights and comments on this manuscript.

\section{Author contributions}

A.V.P., L.A.B. and G.F.S. conceived and designed the experiments. G.F.S. supervised the research. A.V.P. performed all the experiments with the support of L.A.B., L.J. contributed to the hydrogenation and oxygenation experiments and L.M.C.L. contributed to the preparation of the lipid clamping. A.V.P and L.A.B. analyzed the data and constructed the discussion. A.V.P., L.A.B. and G.F.S. wrote the manuscript. All authors read and commented the manuscript. A.V.P. and L.A.B. contributed equally.

\section{Additional information}

Supplementary Information accompanies this paper at https://doi.org/10.1038/s41467018-06608-0
Competing interests: The authors declare no competing interests.

Reprints and permission information is available online at http://npg.nature.com/ reprintsandpermissions/

Publisher's note: Springer Nature remains neutral with regard to jurisdictional claims in published maps and institutional affiliations.

(c) (i) Open Access This article is licensed under a Creative Commons Attribution 4.0 International License, which permits use, sharing, adaptation, distribution and reproduction in any medium or format, as long as you give appropriate credit to the original author(s) and the source, provide a link to the Creative Commons license, and indicate if changes were made. The images or other third party material in this article are included in the article's Creative Commons license, unless indicated otherwise in a credit line to the material. If material is not included in the article's Creative Commons license and your intended use is not permitted by statutory regulation or exceeds the permitted use, you will need to obtain permission directly from the copyright holder. To view a copy of this license, visit http://creativecommons.org/ licenses/by/4.0/.

(C) The Author(s) 2018 